\title{
Seguimiento alejado de pacientes evaluados en una Unidad de Dolor Torácico
}

\author{
LUIGI GABRIELLI, PABLO CASTRO, RAMÓN CORBALÁN, \\ MÓNICA ACEVEDO, PAUL MC-NAB, RICARDO BAEZA, PABLO AGUILERA, \\ MIGUEL MARCHESSE, ALEJANDRO FAJURI, JOSÉ M. MARDONES
}

\section{Long term follow up of patients consulting in a Chest Pain Unit}

Background: Chest pain (CT) constitutes an important cause of consultation and diagnostic dilemma in the emergency room, especially due to the possible presence of coronary disease. Its presentation, diagnosis and prognosis are different between men and women. Aim: To report a follow-up of patients attended at a Chest Pain Unit (CPU), evaluating gender differences. Material and Methods: Prospective evaluation of patients that consulted for chest pain in a period of 4 years. Baseline characteristics and the final diagnosis from CPU or hospitalization were registered. Telephonic follow-up was performed for at least one year. Mortality was determined using the national mortality registry. Results: A total of 1,168 patients aged $62 \pm 23$ years, $69 \%$ men, were followed for a mean of $28 \pm 20$ months. A definitive diagnosis of coronary disease (CD) was done in $32 \%$. Mortality among women and men with $C D$ was 28 and $14 \%$ respectively $(p=0,02)$. Predictor variables for mortality were the presence of a complete left branch block in the initial electrocardiogram, with an odds ratio $(O R)$ of 12,5 (95\% confidence intervals (CI): 1,98-25,8), the presence of coronary disease with an OR of 3,98 (95\% CI: 1,45-13,8) and elevated troponin I with an OR 2,12 (95\% CI: 1,05-7,89). Female gender lost significance in the adjusted model. Conclusions: Complete left branch block, elevated troponin I and coronary etiology of CP are indicators of bad prognosis among patients that consult for CP. Women have twice the mortality of men after 28 months of follow-up.

(Rev Med Chile 2010; 138: 1117-1123).

Key words: Chest pain; Coronary artery diseases; Troponin I.

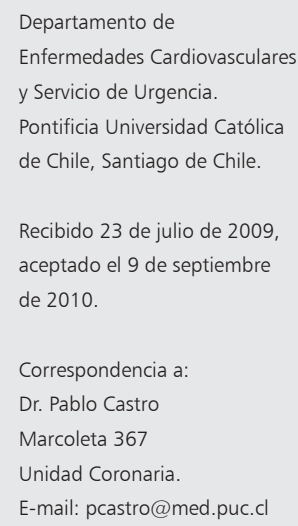

1 dolor torácico (DT) es un síntoma que constituye uno de los más importantes motivos de consulta en los Servicios de Urgencia. En Estados Unidos se registran anualmente alrededor de 6 a 8 millones de consultas, hospitalizándose alrededor de la mitad de los pacientes para evaluación y manejo posterior, de los cuales la mitad corresponden finalmente a síndromes coronarios agudos (SCA $)^{1,2}$. A pesar de las estrategias diagnósticas actuales, se estima que entre 2 y $8 \%$ de los pacientes que se presentan por DT, van a ser enviados a su domicilio con un SCA, doblando la mortalidad asociada de estos pacientes versus aquellos hospitalizados ${ }^{3,4}$. Esto ha ocasionado una sobre hospitalización muchas veces orientada a descartar un probable evento coronario agudo tras una primera evaluación negativa en el Servicio de Urgencia lo que se asocia a costos elevados para los sistemas de salud. Debido a esto y a la variedad de patologías potencialmente graves que subyacen a este síntoma, se han establecido unidades de evaluación denominadas Unidades de Dolor Torácico (UDT) con el fin de: disminuir el error diagnóstico en estos pacientes, establecer una aproximación 
costo-efectiva para este problema, y reducir los tiempos de re-perfusión coronaria en pacientes de alto riesgo $0^{5,6}$. Estas unidades se han desarrollado alrededor del mundo en forma progresiva desde los años 80' con un auge importante en Estados Unidos donde se dispone la mayor cantidad de datos actuales.

Las patologías cardiovasculares y en particular el síndrome coronario agudo (SCA) constituyen la principal causa de mortalidad en nuestro país. Los efectos de un mayor acceso de los pacientes a los Servicios de Urgencia, y las campañas sanitarias nacionales que promueven la consulta precoz frente al DT han generado tensiones sobre el sistema de urgencia para satisfacer la demanda con el menor error posible. Además se han establecido políticas nacionales, que por ley, exigen la atención y diagnóstico oportuno de los pacientes que consultan por DT, por lo que la implementación de UDT se ha convertido cada vez más en una necesidad.

En nuestro país existe escasa experiencia en relación a las Unidades de Dolor Torácico. En agosto de 2002 se creó la primera UDT en nuestro hospital con la primera experiencia publicada al respecto ${ }^{7}$. Nuestra institución es un hospital universitario urbano de alta complejidad, con 80.000 consultas al año en el Servicio de Urgencia y $4 \%$ de las consultas es por DT. La UDT es dirigida por el Departamento de Enfermedades Cardiovasculares y coordinada por nuestro Servicio de Urgencia; ha demostrado ser eficiente para prevenir eventos adversos a corto plazo en pacientes dados de alta desde el Servicio de Urgencia. Sin embargo, el seguimiento a mediano y largo plazo de los pacientes manejados en nuestra UDT aún no ha sido evaluado. Por otro lado, existe evidencia en la literatura de diferencias respecto a la presentación, diagnóstico y pronóstico alejado entre hombres y mujeres que se evalúan en la UDT, destacando entre las mujeres, la variabilidad en los síntomas acompañantes, mayor carga de factores de riesgo y peor pronóstico en aquellas con diagnóstico de enfermedad coronaria (EC) respecto a los hombres $^{8-13}$. Esto tampoco ha sido evaluado en nuestra población de mujeres que consultan por DT.

Los objetivos del presente estudio son evaluar el seguimiento alejado de pacientes que consultan en la UDT con dolor de origen no traumático de un hospital universitario y caracterizar a los pacientes basalmente y en el seguimiento, analizando las diferencias según género.

\section{Pacientes y Métodos}

Estudio prospectivo de pacientes que concurrieron por DT no traumático al Servicio de Urgencia del Hospital Clínico de la Universidad Católica en horario diurno, en el período comprendido entre agosto de 2002 y julio de 2006. La UDT fue organizada dentro del propio Servicio de Urgencia y su funcionamiento, organización y metodología de estratificación de riesgo se describieron con anterioridad ${ }^{7}$. Se registraron antecedentes clínicos, características semiológicas del dolor, alteraciones electrocardiográficas y marcadores de daño miocárdico. Los niveles de troponina I (ensayo AccuTnI de Beckman Coulter tm.) se interpretaron según los límites fijados por el laboratorio local. Un nivel plasmático superior a $0,05 \mathrm{ng} / \mathrm{ml}$ fue considerado como positivo. Se registró, en todos los pacientes, el diagnóstico definitivo al alta hospitalaria o desde la UDT.

Se excluyeron del análisis inicial y del seguimiento a los pacientes con diagnóstico de infarto agudo al miocardio (IAM) con supradesnivel del ST (SDST), tromboembolismo pulmonar y síndromes aórticos agudos.

En todos los pacientes con criterios de riesgo alto e intermedio de SCA, anteriormente definido ${ }^{7}$, se realizó coronariografía; esto también quedaba a criterio del cardiólogo tratante. La presencia de EC se definió con la existencia de al menos una lesión coronaria considerada como significativa por el hemodinamista en el estudio angiográfico.

Todos los pacientes fueron seguidos mediante entrevistas telefónicas por al menos un año. Se registraron nuevas consultas por dolor torácico, hospitalizaciones por eventos cardiovasculares (ECV) y mortalidad general. Ésta última fue corroborada mediante certificado de defunción según la base de datos del Registro Civil. Se consideraron ECV: angina inestable, infarto con SDST e infarto sin SDST. Los ECV fueron corroborados en la ficha clínica.

Las variables continuas se expresaron como promedio \pm DE y las discretas como porcentajes. Las diferencias basales y en el seguimiento según género fueron evaluadas mediante test de chicuadrado y t-Student según correspondía. Además se construyeron curvas de sobrevida con el método de Kaplan-Meier y se evaluaron sus diferencias mediante log rank-test. Las variables predictoras univariadas de muerte o evento cardiovascular se 
incluyeron en un modelo de regresión logística múltiple escalonada para identificar los predictores independientes de un punto final compuesto de muerte o ECV. Las variables predictoras de este punto final compuesto se evaluaron en modelos ajustados y no ajustados. Se consideró significativo un valor $\mathrm{p}<0,05$. Todos los análisis se realizaron con el "software" SPSS 15.0, Illinois, MA USA.

\section{Resultados}

Entre agosto de 2002 y julio de 2006 fueron evaluados en la UDT de nuestro hospital 1.168 pacientes, correspondiendo al $4,8 \%$ de la totalidad de las consultas en el Servicio de Urgencia. Los resultados de alta, hospitalización y diagnóstico de la UDT se describieron con anterioridad ${ }^{7,14}$. Las características demográficas y factores de riesgo del grupo total y según género se muestran en las Tablas 1 y 2 respectivamente. La edad promedio de los pacientes fue de $62 \pm 23$ años, $69 \%$ eran hombres y se hizo el diagnóstico definitivo de EC en el $32,2 \%$ del total de pacientes, en las mujeres se hizo en el 26\% y en los hombres el 34\% ( $\mathrm{p}=$
0,02). Las características semiológicas del dolor, del electrocardiograma y troponina I iniciales según género, se muestran en la Tabla 3.

El seguimiento promedio fue de $28 \pm 20$ meses con una pérdida de $9 \%$ para el registro de eventos, pero sin pérdidas en el seguimiento para mortalidad. La mortalidad general durante el seguimiento fue de $9,2 \%$. Las mujeres con EC presentaron una mortalidad de $27,6 \%$ versus $14 \%$ ( $\mathrm{p}=0,02)$ en los hombres (Figura 1). Los pacientes sin el diagnós-

Tabla 1. Característica demográficas y factores de riesgo del grupo total de pacientes

\begin{tabular}{|ll|}
\hline Número de pacientes & 1.168 \\
\hline Edad \pm DE (años) & $62 \pm 23$ \\
\hline Sexo masculino (\%) & 69 \\
\hline Hipertensión arterial (\%) & 49 \\
\hline Diabetes mellitus tipo 2 (\%) & 15 \\
\hline Dislipidemia (\%) & 39 \\
\hline Tabaquismo (\%) & 33 \\
\hline
\end{tabular}

Tabla 2. Características demográficas y factores de riesgo según género

\begin{tabular}{|lccc|}
\hline & Mujeres & Hombres & p \\
\hline Edad & $68 \pm 13$ & $60 \pm 27$ & $<0,001$ \\
Hipertensión arterial & $59 \%$ & $44 \%$ & $<0,001$ \\
\hline Dislipidemia & $48 \%$ & $32 \%$ & 0,05 \\
Diabetes mellitus & $14 \%$ & $15 \%$ & 0,3 \\
\hline Tabaquismo & $27 \%$ & $34 \%$ & 0,1 \\
Antecedentes coronarios & $6 \%$ & $18 \%$ & 0,01 \\
\hline
\end{tabular}

Antecedentes coronarios: IAM previo, cirugía de revascularización miocárdica previa.

Tabla 3. Características semiológicas, electrocardiográficas y troponina I según género en pacientes que consultan por dolor torácico no traumático

\begin{tabular}{|lccc|}
\hline & Mujeres & Hombres & p \\
\hline Síntomas neurovegetativos & $41 \%$ & $21 \%$ & 0,04 \\
\hline Dolor anginoso típico & $31 \%$ & $59 \%$ & 0,01 \\
\hline ECG con IDST & $6 \%$ & $9 \%$ & 0,04 \\
\hline ECG inespecífico & $22 \%$ & $13 \%$ & 0,01 \\
\hline Troponina I positiva (inicial) & $6 \%$ & $13 \%$ & 0,02 \\
\hline
\end{tabular}


tico de EC presentaron una mortalidad de 4\%, sin diferencia entre hombres y mujeres. Durante el seguimiento $18,5 \%$ de los pacientes presentó un ECV y $28,5 \%$ de los pacientes reconsultaron al menos una vez en la UDT. Los ECV y la nueva consulta en la UDT según género y presencia o ausencia de enfermedad coronaria se muestran en las Figuras 2 y 3.

Los predictores independientes para el punto final compuesto de mortalidad general o ECV, en el
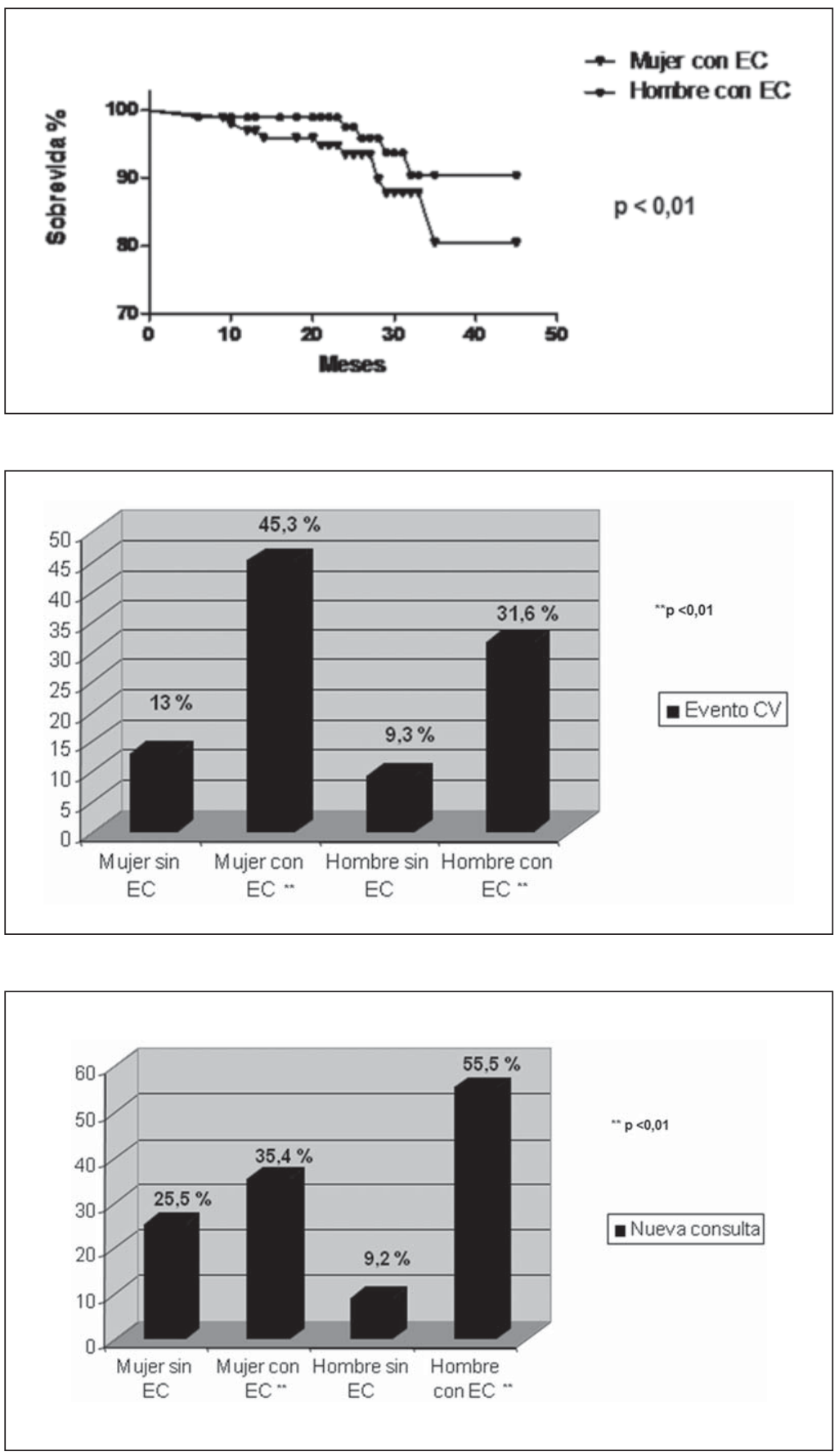

Figura 1. Curva de sobrevida en pacientes con enfermedad coronaria (EC) según género.

Figura 2. Evento cardiovascular al seguimiento según género y diagnóstico EC.

Figura 3. Nueva consulta en la UDT según género y diagnóstico de EC. 

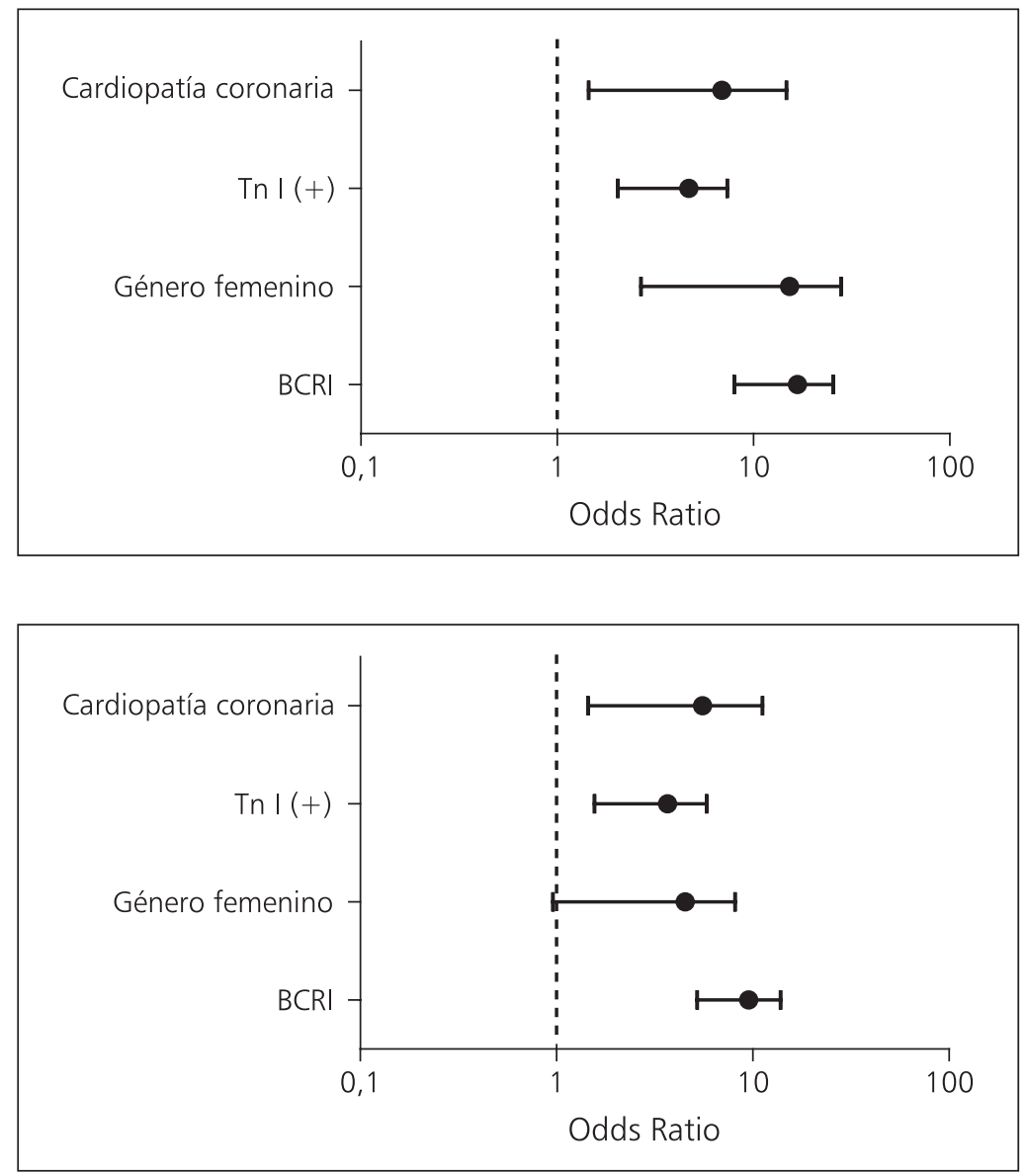

Figura 4. Predictores independientes de mortalidad y evento cardiovascular en modelo no ajustado. modelo no ajustado fueron: bloqueo completo de rama izquierda (BCRI) en el ECG inicial (OR 17,68 $(2,14-36,6))$, género femenino (OR 5,17(1,24$41,55))$, la presencia de enfermedad coronaria (OR 4,55(1,45-14,7)), troponina I elevada (OR $3,17(1,02-9,83))$ (Figura 4). En el modelo ajustado por edad y factores de riesgo tradicionales las variables predictoras fueron: bloqueo completo de rama izquierda en el ECG inicial OR 12,52 $(1,98-25,8)$, la presencia de enfermedad coronaria OR 3,98 (1,45-13,8), troponina I elevada OR 2, 12 $(1,05-7,89)$; el género femenino pierde significado estadístico en el modelo ajustado OR: 1,02 (0,8825,8) (Figura 5).

\section{Discusión}

Existen pocos datos en nuestro medio sobre la caracterización y estratificación de riesgo de pacientes que consultan en el Servicio de Urgencia por $\mathrm{DT}^{14}$, sin existir datos sobre el seguimiento de estos pacientes, correspondiendo la mayoría de los datos a experiencias de centros extranje$\operatorname{ros}^{9,10,15}$.

Los pacientes que consultan por DT y a los cuales se les diagnostica EC tienen una mortalidad elevada en el seguimiento a largo plazo, siendo casi el doble en la población femenina, situación que desde el punto de vista fisiopatológico no está del todo aclarada. Algunas explicaciones para esta importante diferencia estarían dadas por la mayor edad y carga de factores de riesgo que presenta nuestra población femenina con EC, situación que se corrobora en otros registros ${ }^{9,10}$. Por otra parte, dentro del total de pacientes manejados inicialmente como SCA, la población femenina finalmente presenta menos marcadores de daño miocárdico positivos y menor porcentaje de 
lesiones coronarias significativas respecto de la población masculina lo que muchas veces puede retrasar y subutilizar las terapias probadas para el SCA en aquellas pacientes con $\mathrm{EC}^{16}$. Además se puede observar dentro de los pacientes con EC, una significativa menor reconsulta por DT en las mujeres respecto de los hombres, lo que sumado a la mayor frecuencia de manifestaciones atípicas podrían explicar una mala interpretación y conductas erradas, tanto por parte de las pacientes como de los médicos que evalúan el DT lo que estaría incidiendo finalmente en el peor pronóstico de la población femenina. Esta última situación incluso se da en pacientes con EC conocida en donde la población femenina recibe hasta el doble de prescripción antidepresiva y ansiolítica respecto de la población masculina ${ }^{17}$.

Otro punto importante a mencionar es el buen pronóstico de los pacientes que consultan por DT que no se les hace finalmente el diagnóstico de EC, los cuales tienen una mortalidad menor al 5\% en el seguimiento, siendo esto similar a lo reportado por otras experiencias ${ }^{18}$. Sin embargo, dentro de la población femenina existe un subgrupo de pacientes sin EC significativa que persisten con DT que tienen mayores eventos en el seguimiento a largo plazo respecto de la población asintomática ${ }^{19,20}$.

En el presente estudio, los predictores de mortalidad alejada y ECV más importantes en los pacientes que consultan por DT fueron: presencia de BCRI en el ECG inicial, presencia de marcadores de daño miocárdico y la etiología coronaria del DT, todos elementos que se corroboran en otros sistemas de puntaje pronóstico en el contexto de $\mathrm{SCA}^{14}$. El género femenino como factor pronóstico al ser ajustado por edad y factores de riesgo pierde su valor. Toda esta información muestra la importancia del estudio diferenciado del DT en la mujer, sobre todo por la ominosa evolución alejada de las pacientes con EC, la cual muy probablemente está influenciada por la mayor edad de esta población, el manejo de sus factores de riesgo y el reconocimiento oportuno de cuadros coronarios agudos en el contexto de la evaluación del DT.

Este estudio tiene las limitaciones propias de un registro y la pérdida del seguimiento de un porcentaje de pacientes, sin embargo, respecto al dato de mortalidad entrega una importante información respecto a la evolución de pacientes con EC.

\section{Referencias}

1. Mc Caig LF, Burt CW. National hospital Ambulatory Medic al Care Survey: 2002 Emergency Department Summary. Advance Data from Vital and Health Statistics Publication No: 335. Hyattsville, Md, National Center for Health Statistics, 2004.

2. Pope JH, Selker HP: Diagnosis of acute cardiac isquemia. Emerg Med Clin North Am 2003; 21: 27-59.

3. Lau J, Ioannidis JP, Balk EM, Milch C, Terrin N, Chew PW, et al. Diagnosing acute cardiac ischemia in the emergency department: a systematic review of the accuracy and clinical effect of current technologies. Ann Emerg Med 2001; 37: 453-60.

4. Pope JH, Aufderheide TP, Ruthazer R, Woolard RH, Feldman JA, Beshansky JR, et al. Missed diagnoses of acute cardiac ischemia in the emergency department. $\mathrm{N}$ Engl J Med 2000; 20; 342: 1163-70.

5. Graff LG, Dallara J, Ross MA, Joseph AJ, Itzcovitz J, Andelman RP, et al. Impact on the care of the emergency department chest pain patient from the chest pain evaluation registry (CHEPER) study. Am J Cardiol 1997; 1; 80: 563-8.

6. Bassan R, Gibler W. Unidades de dolor torácico: estado actual del paciente con dolor torácico en los servicios de urgencias. Rev Esp Cardiol 2001; 54: 1103-9.

7. Castro P, Corbalán R, Isa R, Gabrielli L, Pérez O, Chamorro $\mathrm{G}$, et al. Unidad de dolor torácico: primera experiencia en Chile. Rev Med Chile 2007; 135: 839-45.

8. Krantz DS, Olson MB, Francis JL, Phankao C, Bairey Merz CN, Sopko G, et al. Anger, hostility, and cardiac symptoms in women with suspected coronary artery disease: the Women's Ischemia Syndrome Evaluation (WISE) Study. J Womens Health (Larchmt) 2006; 10: 1214-23.

9. Shaw LJ, Bairey Merz CN, Pepine CJ, Reis SE, Bittner V, Kelsey SF, et al. Insights from the NHLBI-Sponsored Women's Ischemia Syndrome Evaluation (WISE) Study: Part I: gender differences in traditional and novel risk factors, symptom evaluation, and gender-optimized diagnostic strategies. J Am Coll Cardiol 2006; 47 (3 Suppl): S4-S20.

10. Bairey Merz CN, Shaw LJ, Reis SE, Bittner V, Kelsey SF, Olson $\mathrm{M}$, et al. Insights from the NHLBI-Sponsored Women's Ischemia Syndrome Evaluation (WISE) Study: Part II: gender differences in presentation, diagnosis, and outcome with regard to gender-based pathophysiology of atherosclerosis and macrovascular and microvascular coronary disease. J Am Coll Cardiol 2006; 47 (3 Suppl): S21-9.

11. Pepine CJ. Ischemic heart disease in women. J Am Coll 
Cardiol 2006; 47 (3 Suppl): S1-3.

12. Merz NB, Johnson BD, Kelsey PSF, Reis SE, Lewis JF, Reichek N, et al; Wise Study Group. Women's Ischemia Syndrome Evaluation. Diagnostic, prognostic, and cost assessment of coronary artery disease in women. Am J Manag Care 2001; 10: 959-65.

13. Olson MB, Kelsey SF, Matthews K, Shaw LJ, Sharaf BL, Pohost GM, et al. Symptoms, myocardial ischaemia and quality of life in women: results from the NHLBIsponsored WISE Study. Eur Heart J 2003; 16: 1506-14.

14. Gabrielli LA, Castro PF, Verdejo HE, McNab PA, Llevaneras SA, Mardonez JM, et al. [Predictors of acute coronary syndrome without ST segment elevation and risk stratification in the chest pain unit]. Rev Med Chile 2008; 136: 442-50. Epub 2008 Jun 16.

15. Pastor Torres LF, Pavón-Jiménez R, Reina Sánchez M, Caparros Valderrama J, Mora Pardo JA. [Chest pain unit: one-year follow-up.]. Rev Esp Cardiol 2002; 10: 1021-7.

16. Shaw LJ, Shaw RE, Merz CN, Brindis RG, Klein LW, Nallamothu B, et al. Impact of ethnicity and gender differences on angiographic coronary artery disease prevalence and in-hospital mortality in the American College of Cardiology-National Cardiovascular Data Registry. Circulation 2008; 117: 1787-801. Epub 2008
Mar 31.

17. Gopalakrishnan P, Ragland MM, Tak T. Gender differences in coronary artery disease: review of diagnostic challenges and current treatment. Postgrad Med 2009; 12: 60-8.

18. Zarauza J, Rodríguez-Lera MJ, Ceballos B, Piedra L, Dierssen T, Pérez J. [Follow-up findings one year after discharge from a chest pain unit]. Rev Esp Cardiol 2003; 56: 1137-40.

19. Johnson BD, Shaw LJ, Pepine CJ, Reis SE, Kelsey SF, Sopko G, et al. Persistent chest pain predicts cardiovascular events in women without obstructive coronary artery disease: results from the NIH-NHLBI-sponsored Women's Ischaemia Syndrome Evaluation (WISE) study. Eur Heart J 2006; 27: 1408-15. Epub 2006 May 23.

20. Johnson BD, Shaw LJ, Buchthal SD, Bairey Merz CN, Kim HW, Scott KN, et al; National Institutes of HealthNational Heart, Lung, and Blood Institute. Prognosis in women with myocardial ischemia in the absence of obstructive coronary disease: results from the National Institutes of Health-National Heart, Lung, and Blood Institute-Sponsored Women's Ischemia Syndrome Evaluation (WISE). Circulation 2004; 109: 2993-9. Epub 2004 Jun 14. 